\title{
Searching Grade Scheme and Malware Recognition Within Google Play Application
}

\author{
Karthika. A
}

ME Scholar, Computer Science and Engineering, Velalar College of Engineering and Technology, Erode, Tamilnadu, India

\begin{abstract}
To introduce FairPlay, a work of fiction system that discover and leverages traces left behind by fraudsters, to distinguish both malware and apps subjected to investigate status fraud. FairPlay associate review behavior and distinctively combine detect review associations with linguistic and behavioral signals gleaned from Google Play app records (87 K apps, $2.9 \mathrm{M}$ reviews, and $2.4 \mathrm{M}$ reviewers, unruffled over half a year), in order to organize suspicious apps. FairPlay achieves over 95 percent accuracy in classify gold regular datasets of malware, counterfeit and legitimate apps. Deceptive behaviors in Google Play, the most trendy Android app market, fuel Search rank abuse and malware proliferation. To make out malware, preceding work has paying attention on app executable and acquiescence analysis. It will show that 75 percent of the acknowledged malware apps engage in hunt rank fraud. FairPlay discover hundreds of fraudulent apps that presently evade Google Bouncer's recognition machinery.
\end{abstract}

Keywords : Fair Play, Dataset, Google Play, Malware, Google Bouncer, Proliferation.

\section{INTRODUCTION}

Android does a software horde embrace not only in commission organization but also middleware as well as solution applications. Android come in the midst of an Android market which is online software accumulate. It was residential by Google. It allows robot users to select, and download applications residential by third gathering developers and use them. Android at the start came into subsistence with the sure fire scheme that developments are given the power and autonomy to create engrossing Mobile application while taking advantage of the whole thing that the mobile handset has to offer.

Machine is built on open Linux Kernel. This particular software for Mobile Application is made to be open source, thereby giving the occasion to the developers to commence and incorporate any technological advancement. Fabricate on custom virtual machine android gives its users the totaling usage and purpose power, to begin an interactive and efficient application and prepared Software for your phone. Google's mobile operating device, the robot is its tremendous creation in the perfect creation of Software Applications for the transportable phone arena it also facilitate the g-juice in your transportable thus initiate a whole new world of Mobile Technology skill by its regulars. Arokia IT is technically equipped to initiate any level of these wonderful software applications using the android intellect from Google. Around in the year 2007, Google announce its Android Operating System and Open Handset Alliance through these two major donations to the mobile industry that ultimately misrepresented our knowledge with portable interface. 


\section{LITERATURE REVIEW}

\section{a) GRAPH BASED OPINION SPAM DETECTION}

Graph base approach has been projected to tackle judgment spam. Ye and Akoglu compute the ability of a product to be a spam drive target, and then gather spammers on a 2-hop sub chart induced by the products with the peak chance values. Akoglu et al. Surround fraud detection as a signed network arrangement problem and organize users and harvest that form a bipartite complex, using a propagationbased algorithm.

FairPlay's relational advance differs as it identifies apps reviewed in a bordering time interval, by group of users with a history of reviewing apps in frequent. FairPlay combine the results of this move toward with behavioral and linguistic clues, extract from longitudinal app figures, to perceive mutually investigate rank design and malware apps. We accentuate that search rank fraud goes ahead of estimation spam, as it imply fabricate not only reviews, but also customer app install measures and ratings.

\section{b) AN ANDROID-BASED MECHANISM FOR ENERGY EFFICIENT LOCALIZATION DEPENDING ON INDOOR/OUTDO}

Location-based application on modern smart phone have acknowledged rife usage in today's society-to the point where it can even be said that many have grow to be conditional on these type of applications. Location in turn is used to geotag posts on social medium websites, to distribute the local withstand and news, to help users take the helm to a desired location, and to provide information on close to restaurant and stores. However, users regularly have to balance the expediency and functionality of these location-based applications with a Smartphone's battery life. The tradeoff between these two comes down to exactness against energy. Applications that require fine-grained setting in turn opt to use the power-hungry GPS, while applications with more coarse necessities may use the network-based provider, which is less accurate, but has superior energy savings. The user is often given the ability to toggle position services on or off and, with Android phones, can also selectively allow or disable the formerly mentioned two methods to fine-tune their phone's accuracy/energy transaction. However, in most cases, the regular user will not pay much attention to this option due to absentmindedness, not knowing such options are available, or a lack of familiarity on the liveliness costs. On the other hand, developers of location-based application can trim down energy consumption by smartly choosing among using the GPS or the set-up contributor depending on application rations or other context. However, developers cannot always predict when to energetically control between methods. In other cases, locations return by the network-based method will not be accurate enough for proper functionality of some applications (e.g., navigational applications) - in this case, the GPS will always be invoked regardless of environment or context. As a result, this leads to a waste of energy in situations where the GPS is unavailable or inaccurate, such as in indoor environment or "urban canyons."

\section{c) SEMANTICS-BASED ONLINE MALWARE DETECTION: TOWARDS EFFICIENT REAL- TIME PROTECTION AGAINST MALWARE}

In this work, we in attendance the first system call based come up to using hardware-enhanced planning that employ appliance learning practice for malware recognition. We name it GuardOL (guard online). GuardOL use a novel regularity centralized model (FCM) for attribute structure to learn the malevolent behavioral pattern from known malware sample. Our frequency-centralized model takes the occurrence of resource-critical system calls into account and constructs facial appearance by federation system 
calls using inclusive rules to imprison the semantics of malicious behavior. To this end, GuardOL extract the structure calls (with their point of view and go again values) for the duration of execution on the supercomputer and groups relevant system calls to construct features using FCM. The skin texture obtained from the malware and benign sample are used to educate multilayer perception (MLP), an reproduction neural complex model, which is used at runtime to act upon the arrangement of the running curriculum as malware or benevolent. We widen an architectural design of GuardOL based on our proposed methodology. For the proof of concept, we put into operation our model in FPGA. We leverage the advantages of FPGA platform to obtain a high routine training and exposure at a low cost and reconfigurability for place fabrication functionality upgrade to adapt to new malware samples. Reconfigurability of FPGA also allows sharing of hardware for classifier schooling and runtime exposure. Our draw near aims to capture the semantics of malicious behavior with the proposed frequency federal model with therefore has the potential to detect malware variants with even zeroday (previously unseen) attacks. Compare to the software approaches, GuardOL is resistant to aforesaid advanced malware technique, as it is based on hardware. Unlike software techniques, it cannot be disabling or open by sophisticated technique (e.g., anti-virtual contraption and anti-debugger) and thus malicious activities force not is hidden beginning detection. In addition, our implementation offers a power efficient malware detection design, which has low reserve demands and unimportant piece overhead on the system. The FPGA accomplishment of GuardOL consumes $0.36 \mathrm{~W}$ during training and $0.264 \mathrm{~W}$ at runtime. Our fallout shows that GuardOL achieves faster detection with zero routine penalties on the supercomputer.

\section{d) ANDROID MALWARE DETECTION WITH CONTRASTING PERMISSION PATTERNS}

An anomaly uncovering technique uses its knowledge of what constitute normal behavior to decide the maliciousness of an submission under check up. Instead, misuse detection uses the categorization of what is known to be malicious to settle on the cruelty of an application under inspection. Typically, each routine uses a "summary" in place of the distinctiveness of normal/abnormal application for detecting malware. More exclusively, normal shape is used in anomaly recognition, whereas anomalous profile is old in misuse detection. Generally, anomaly exposure and use wrongly revealing may have sky-scraping false affirmative rate and high false negative rate correspondingly due to the limitations. There is a considerable difference between malwares and clean application on the distribution of dism. Most malwares have much greater dism than that of clean applications, which implies that dism is a perfect metric for distinguishing malwares from clean applications. However, as the consequence of classifier imbalance, almost all instances have positive ethics of dism. To offset this movement away, a positive discrimination coefficient $\varepsilon$, is needed for making the final decisions. Divergent patterns as well as their support degrees of malware and clean datasets are considered as the distinctiveness that discriminates malwares from clean applications. Hence, we present a malware exposure support with a amalgam profile composed by MP, CIP and CoP, which are represented by unique acquiescence patterns in malware dataset, unique permission patterns in unsoiled dataset and frequently required permission pattern respectively. To execute the proposed outline, an ensemble classifier, Enclamald, is developed. Each divergent permission model plays the role of a weak classifier and votes for the ultimate result. We present a metric, dism, as the aggregate result of all involved weak classifiers to quantify how likely a function is malevolent. 


\section{METHODOLOGY}

FairPlay correlate review proceedings and distinctively combine detect appraise interaction with syntactical and behavioral signal gleaned from Google Play app data, in order to identify disbelieving apps. FairPlay achieves over 95\% accuracy in classify gold ordinary datasets of malware, fraudulent and lawful apps. We show that $75 \%$ of the identified malware apps fit into place in search rank fraud. Impartiality discovers hundreds of fraudulent apps that currently evade Google Bouncer's uncovering equipment. FairPlay also help the discovery of more than 1,000 reviews, reported for 193 apps that reveal a new brand of influential review operation. We uncover these malicious acts by picking out such trails. For illustration, the high price tag of setting up valid Google Play financial statement services fraudsters to reprocess their financial statement transversely review writing jobs, assembly them likely to review more apps in familiar than regular users. Resource constraint can compel fraudsters to post reviews surrounded by tiny time interval.

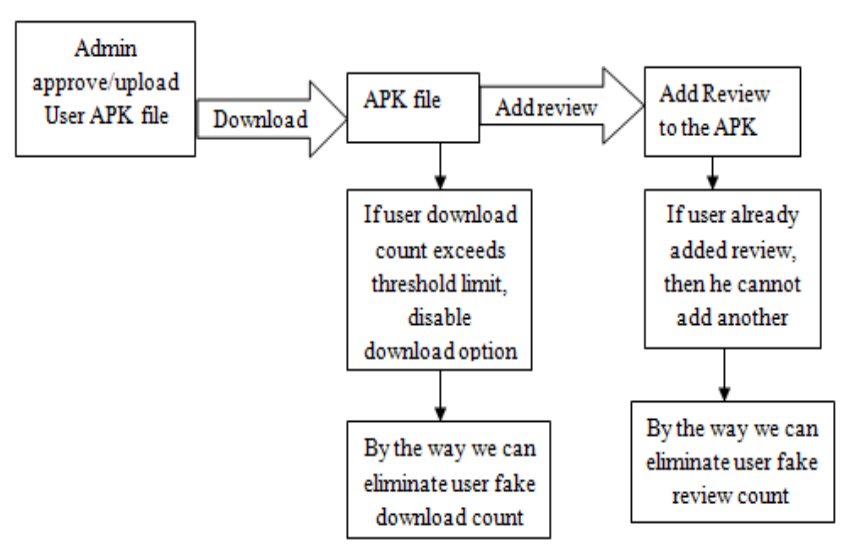

Fig 1. System Architecture

Within this, we inaugurate FairPlay, a narrative organism that discover and leverages traces left following by fraudsters, to perceive both malware and apps subjected to investigate rank fraud. Objectivity show a relationship appraise activities and outstandingly combine detected review relations with syntactical and behavioral signals gleaned from Google Play app data, in order to identify doubtful apps. FairPlay achieves over $95 \%$ accuracy in classifying gold standard datasets of malware, fraudulent and equitable apps. We show that $75 \%$ of the branded malware apps employ in search status deception. FairPlay discover hundreds of fraudulent apps that at this time evade Google Bouncer's detection technology. FairPlay also help the discovery of more than 1,000 reviews, report for 193 apps that divulge a new category of forceful review operation. We unearth these malicious acts by preference out such trails. For instance, the high cost of situation up valid Google Play financial statement forces fraudsters to reuse their balance sheet across assessment writing jobs, making them likely to review more apps in universal than ordinary users. Resource constraints container make fraudsters to situation reviews contained by short time intervals. The 1,024 coerced reviews are posted for 193 apps. While a large amount of the 193 apps have customary less than 20 coerced reviews, 5 apps have every one received more than 40 such reviews. We have observed several duplicate among the coerced reviews. We identify two promising explanation. First, as we earlier mentioned, some apps do not keep track of the user having reviewed them, thus repetitively coerce subsequent review from the equivalent user. A second enlightenment is that seemingly coerced reviews can also be posted as parts of a negative consider rank fraud battle. However, in cooperation scenario describe apps likely to have been subjected to falsified behaviors.

\section{Advantages}

Be capable of detect valid reviews.

$>$ Be able to identify fraud users and malware meter.

Identifies authoritative reviews course of action. 


\section{RESULT AND DISCUSSION}

In order to find confirmation of systematic coercive review campaign, we have parsed the 2.9 million reviews of our dataset to identify those whose text contains one of the root words $1 / 2$ "make", "ask", "force" and "rate". Upon manual scrutiny of the results, we have found 1,024 coerced reviews. The reviews make public that apps involved in coercive review campaign either have bugs (e.g., they ask the user to rate 5 stars even after the user has rated them), or wage the user by removing ads, providing supplementary features, unlocking the next game level, boosting the user's entertainment level or openhanded game point.

The 1,024 coerced reviews were posted for 193 apps. While most of the 193 apps have received less than 20 coerced reviews, 5 apps have each routine more than 40 such reviews. We have pragmatic several duplicate surrounded by the coerce review. We identify two promising explanations. First, as we previously mention, some apps carry out not keep track of the user having reviewed them, thus repeatedly coerce subsequent reviews starting the same user. A second explanation is that seemingly coerced reviews, can also posted as part of a offputting search rank fraud operation. However, both scenarios describe apps likely to have be subjected to deceptive behaviors.

\section{REFERENCES}

[1]. S. Mlot. (2014, Apr. 8). "Top Android App a Scam, Pulled From Google Play," PCMag. Available:

http://www.pcmag.com/article2/0,2817,245616 5,00.asp

[2]. D. Roberts. (2015, Jul. 8). "How to spot fake apps on the Google Play store," Fortune. Available: http://fortune.com/2015/07/08/google-playfake-app/

[3]. I.Burguera, U. Zurutuza, and S. Nadjm-Tehrani, "Crowdroid: Behavior based malware detection system for Android," in Proc.ACM SPSM, 2011, pp. 15-26

[4]. S. Yerima, S. Sezer, and I. Muttik, "Android Malware detection using parallel machine learning classifiers," in Proc. NGMAST, Sep. 2014, pp. 37-42.

[5]. Alfonso Munoz, Ignacio Mart '1n, Antonio Guzman, Jos ' e Alberto Hern ' andez, IEEE Android malware detection from Google Play meta-data: Selection of important features.2015, pages,245-251]Alfonso Munoz, Ignacio Mart 'in, Antonio Guzman, Jos ' e Alberto Hern ' andez, IEEE Android malware detection from Google Play meta-data: Selection of important features.2015, pages,245251

[6]. Chia-Mei Chen, Je-Ming Lin, Gu-Hsin Lai,IEEE Detecting Mobile Application Malicious Behaviors Based on Data Flow of Source Code.2014 International Conference on Trustworthy Systems and their Applications pp 95-109

[7]. E.-P. Lim, V.-A. Nguyen, N. Jindal, B. Liu, and H. W. Lauw. Detecting product review spammers using rating behaviors. In Proceedings of the 19th ACM international conference on Information and knowledge management, CIKM '10, pages 939-948, 2010.

Cite this article as :Karthika. A, "Searching Grade Scheme and Malware Recognition Within Google Play Application", International Journal of Scientific Research in Science and Technology (IJSRST), Online ISSN : 2395-602X, Print ISSN : 2395-6011, Volume 6 Issue 2, pp. 08-12, March-April 2019. Available at doi : $\quad$ https://doi.org/10.32628/IJSRST196177 Journal URL : http://ijsrst.com/IJSRST196177 